\title{
GROUPS OF INFINITE RANK IN WHICH NORMALITY IS A TRANSITIVE RELATION
}

\author{
M. DE FALCO, F. DE GIOVANNI, C. MUSELLA \\ Dipartimento di Matematica e Applicazioni, Università di Napoli Federico II Complesso \\ Universitario Monte S. Angelo, Via Cintia, I-80126 Napoli, Italy \\ e-mail: \{degiovan, mdefalco,cmusella\}@unina.it \\ and Y. P. SYSAK \\ Institute of Mathematics, Ukrainian National Academy of Sciences vul. Tereshchenkivska 3, \\ 01601 Kiev, Ukraine \\ e-mail:sysak@imath.kiev.ua
}

(Received 19 October 2012; accepted 3 April 2013; first published online 30 August 2013)

\begin{abstract}
A group is called a $T$-group if all its subnormal subgroups are normal. It is proved here that if $G$ is a periodic (generalized) soluble group in which all subnormal subgroups of infinite rank are normal, then either $G$ is a $T$-group or it has finite rank. It follows that if $G$ is an arbitrary group whose Fitting subgroup has infinite rank, then $G$ has the property $T$ if and only if all its subnormal subgroups of infinite rank are normal.
\end{abstract}

2000 Mathematics Subject Classification. 20E15.

1. Introduction. A group $G$ is called a $T$-group if normality in $G$ is a transitive relation, i.e. if all subnormal subgroups of $G$ are normal. The structure of soluble $T$-groups has been described by Gaschütz [11] in the finite case and by Robinson [12] for arbitrary groups. It turns out in particular that soluble groups with the property $T$ are metabelian and hypercyclic, and that finitely generated soluble $T$-groups are either finite or abelian. More recently, many authors have investigated soluble groups in which normality is imposed only to certain relevant systems of subnormal subgroups (see, for instance, $[\mathbf{3}, \mathbf{4}, \mathbf{1 0}]$ ), and in most cases it turns out that if such groups do not have the property $T$, then they cannot be too large.

Recall that a group $G$ is said to have finite (Prüfer) rank $r$ if every finitely generated subgroup of $G$ can be generated by at most $r$ elements, and $r$ is the least positive integer with such a property. The investigation of the influence on a (generalized) soluble group of the behavior of its subgroups of infinite rank has been developed in a series of recent papers (see, for instance, $[\mathbf{1}, \mathbf{2}, \mathbf{6}, \mathbf{7}, \mathbf{8}, \mathbf{1 4}])$. In particular, Evans and Kim [9] have proved that if $G$ is a (generalized) soluble group in which all subgroups of infinite rank are normal, then either $G$ is a Dedekind group or it has finite rank.

The aim of this paper is to provide further contributions to this topic, studying groups in which all subnormal subgroups of infinite rank are normal. In fact, our main result deals with periodic groups with such a property.

Theorem A. Let $G$ be a periodic hyperabelian group whose subnormal subgroups of infinite rank are normal. Then either $G$ has finite rank or it is a T-group. 
Recall here that a group is called hyperabelian if it has an ascending normal series with abelian factors. Suitable examples will show that Theorem A cannot be extended to the case of non-periodic groups. On the other hand, for arbitrary groups the situation is clear at least when the Fitting subgroup (i.e. the subgroup generated by all nilpotent normal subgroups) has infinite rank. This is proved by our second main result.

Theorem B. Let $G$ be a group whose subnormal subgroups of infinite rank are normal. If the Fitting subgroup of $G$ has infinite rank, then $G$ is a T-group.

Most of our notation is standard and can be found in [13].

2. Proofs and examples. It is known that in any $T$-group every nilpotent normal subgroup centralizes the commutator subgroup. Our next two lemmas show that, in the case of a group $G$ in which all subnormal subgroups of infinite rank are normal, this holds at least for nilpotent normal subgroups of infinite rank, and actually $G$ is a $T$-group, provided that it contains such a subgroup.

LEMMA 1. Let $G$ be a group whose subnormal subgroups of infinite rank are normal, and let $N$ be a nilpotent normal subgroup of $G$. If $N$ has infinite rank, then all subgroups of $N$ are normal in $G$. In particular, $N$ is a Dedekind group and $\left[N, G^{\prime}\right]=\{1\}$.

Proof. Since each subgroup of $N$ is subnormal in $G$, we have that all subgroups of infinite rank of $N$ are normal in $G$, and in particular $N$ is a Dedekind group (see [9, Theorem C]). Then $N$ contains an abelian subgroup $A$ of infinite rank which is the direct product of cyclic subgroups. Let $x$ be any element of $N$ so that there exists a subgroup $B$ of infinite rank of $A$ such that $B \cap\langle x\rangle=\{1\}$. Consider the set $\mathfrak{L}$ of all subgroups of finite index of $B$. For each element $L$ of $\mathfrak{L}$, the product $L\langle x\rangle$ has infinite rank, and so it is normal in $G$. Therefore, the subgroup

$$
\langle x\rangle=\bigcap_{L \in \mathfrak{L}} L\langle x\rangle
$$

is also normal in $G$. It follows that all subgroups of $N$ are normal in $G$. Thus, the factor group $G / C_{G}(N)$ is isomorphic to a group of power automorphisms of $N$, and hence it is abelian so that $G^{\prime} \leq C_{G}(N)$.

LEMMA 2. Let $G$ be a group whose subnormal subgroups of infinite rank are normal. If the centralizer $C_{G}\left(G^{\prime}\right)$ has infinite rank, then $G$ is a T-group.

Proof. As $C_{G}\left(G^{\prime}\right)$ is nilpotent, it follows from Lemma 1 that all subgroups of $C_{G}\left(G^{\prime}\right)$ are normal in $G$. Let $X$ be any subnormal subgroup of finite rank of $G$. Then $C_{G}\left(G^{\prime}\right)$ contains a subgroup $A$ of infinite rank, which is the direct product of cyclic subgroups such that $A \cap X=\{1\}$. Let $\mathfrak{L}$ be the set of all subgroups of finite index of $A$. For each element $L$ of $\mathfrak{L}$, the product $L X$ is a subnormal subgroup of infinite rank of $G$ so that $L X$ is normal in $G$, and hence the subgroup

$$
X=\bigcap_{L \in \mathfrak{L}} L X
$$

is also normal in $G$. Therefore, $G$ is a $T$-group.

COROLlaRY 3. Let $G$ be a group whose subnormal subgroups of infinite rank are normal. If $G$ contains a nilpotent normal subgroup of infinite rank, then $G$ is a T-group. 
Proof. Let $N$ be a nilpotent normal subgroup of infinite rank of $G$. Then $N$ is contained in the centralizer of $G^{\prime}$ by Lemma 1 so that $C_{G}\left(G^{\prime}\right)$ has infinite rank and $G$ is a $T$-group by Lemma 2.

LEMMA 4. Let $G$ be a periodic group of infinite rank whose subnormal subgroups of infinite rank are normal, and let $N$ be a normal subgroup of $G$ contained in $G^{\prime}$. If $G^{\prime} / N$ is a Černikov group, then $G / N$ is a $T$-group.

Proof. The factor group $G / C_{G}\left(G^{\prime} / N\right)$ is isomorphic to a periodic group of automorphisms of $G^{\prime} / N$, and hence it is a Cernikov group (see [13, Part 1, Theorem 3.29]). Thus, the centralizer $C_{G}\left(G^{\prime} / N\right)$ has infinite rank, and it follows from Lemma 2 that $G / N$ is a $T$-group.

It is well known that any maximal abelian normal subgroup of a nilpotent group is self-centralizing. Our next result shows that a similar property holds in hyperabelian groups for maximal nilpotent normal subgroups with class at most 2.

LeMma 5. Let $G$ be a hyperabelian group, and let $N$ be a maximal element of the set of all nilpotent normal subgroups of $G$ with class at most 2. Then $C_{G}(N)=Z(N)$.

Proof. Assume for a contradiction that $C_{G}(N) \neq Z(N)$. As $G$ is hyperabelian, there exists an abelian nontrivial normal subgroup $A / Z(N)$ of $G / Z(N)$ such that $A \leq C_{G}(N)$. Then $A^{\prime}$ is contained in $Z(N)$, and hence

$$
(A N)^{\prime}=A^{\prime} N^{\prime}[A, N]=A^{\prime} N^{\prime} \leq Z(N) \leq Z(A N) .
$$

Therefore, the normal subgroup $A N$ of $G$ is nilpotent with class at most 2 so that $A N=N$ and $A$ is contained in $N$. This contradiction shows that $C_{G}(N)=Z(N)$.

LemMA 6. Let $G$ be a periodic hyperabelian group of infinite rank in which all subnormal subgroups of infinite rank are normal. Then $G$ is soluble.

Proof. Let $N$ be a maximal element of the set of all nilpotent normal subgroups of $G$ with class at most 2. If $N$ has infinite rank, it follows from Corollary 3 that $G$ is a $T$-group, and so it is metabelian. Suppose now that $N$ has finite rank. For each prime number $p$, the unique Sylow $p$-subgroup $N_{p}$ of $N$ is a Černikov group, and hence the factor group $G / C_{G}\left(N_{p}\right)$ is finite (see [13, Part 1, p. 85]). Thus, $G / C_{G}\left(N_{p}\right)$ is a soluble $T$-group, and so it is metabelian. As

$$
C_{G}(N)=\bigcap_{p} C_{G}\left(N_{p}\right)
$$

it follows that $G / C_{G}(N)$ is metabelian. On the other hand, $C_{G}(N)=Z(N)$ by Lemma 5 , and hence $G$ is soluble.

Our next two results deal with the special cases of Theorem $\mathrm{A}$ in which the commutator subgroup is abelian and nilpotent respectively.

LEMMA 7. Let G be a periodic metabelian group of infinite rank in which all subnormal subgroups of infinite rank are normal. Then $G$ is a T-group.

Proof. By Lemma 2 we may suppose that the centralizer $C_{G}\left(G^{\prime}\right)$ has finite rank so that, in particular, the commutator subgroup $G^{\prime}$ has finite rank. For each prime 
number $p$, write

$$
G^{\prime}=K_{p} \times K_{p^{\prime}}
$$

where $K_{p}$ and $K_{p^{\prime}}$ are the $p$-component and the $p^{\prime}$-component of $G^{\prime}$ respectively. As $G^{\prime} / K_{p^{\prime}} \simeq K_{p}$ is a Černikov group, it follows from Lemma 4 that the factor group $G / K_{p^{\prime}}$ is a $T$-group. Let $x$ be any element of $G^{\prime}$ of prime-power order $p^{n}$. The subnormal subgroup $\langle x\rangle K_{p^{\prime}}$ is normal in $G$, and it follows that $\langle x\rangle$ is normal in $G$, as the normal closure $\langle x\rangle^{G}$ is a $p$-group. Therefore, all subgroups of $G^{\prime}$ are normal in $G$.

Let $X$ be any subnormal subgroup of finite rank of $G$, and consider the normal subgroup $X \cap G^{\prime}$ of $G$. In order to prove that $X$ is normal in $G$, we may replace $G$ by $G / X \cap G^{\prime}$, and hence suppose that $X \cap G^{\prime}=\{1\}$. Then $X$ is abelian, and it is enough to show that all its primary components are normal in $G$ so that it can also be assumed without loss of generality that $X$ is a $p$-group for some prime number $p$. In the factor group $\bar{G}=G / K_{p}$, the subnormal subgroup $\bar{X}$ is a Sylow $p$-subgroup of $\bar{X} \bar{G}^{\prime}$ so that it is normal in $\bar{G}$, and hence $X K_{p}$ is a normal subgroup of $G$. On the other hand, the factor group $G / K_{p^{\prime}}$ is a $T$-group, and so also $X K_{p^{\prime}}$ is normal in $G$. It follows that

$$
X=X K_{p} \cap X K_{p^{\prime}}
$$

is normal in $G$. Therefore, $G$ is a $T$-group.

LEMMA 8. Let $G$ be a periodic group of infinite rank in which all subnormal subgroups of infinite rank are normal. If the commutator subgroup $G^{\prime}$ of $G$ is nilpotent, then $G$ is a T-group.

Proof. Assume for a contradiction that $G$ is not a $T$-group so that by Lemma 7 the subgroup $G^{\prime}$ cannot be abelian. Then it follows from Lemma 1 that $G^{\prime}$ has finite rank. For each prime number $p$ we have

$$
G^{\prime}=K_{p} \times K_{p^{\prime}}
$$

where $K_{p}$ is a $p$-group and $K_{p^{\prime}}$ is a $p^{\prime}$-group. As $G^{\prime} / K_{p^{\prime}} \simeq K_{p}$ is a Černikov group, Lemma 4 yields that $G / K_{p^{\prime}}$ is a $T$-group, and, in particular, it is metabelian. On the other hand,

$$
\bigcap_{p} K_{p^{\prime}}=\{1\}
$$

and hence $G$ is metabelian. This contradiction proves the lemma.

We are now ready to complete the proof of our main statement.

Proof of Theorem A. Assume for a contradiction that the statement is false. In particular, the group $G$ has infinite rank and so it is soluble by Lemma 6. Thus, the counterexample $G$ can be chosen with a minimal derived length. Let $A$ be the smallest nontrivial term of the derived series of $G$. It follows from Corollary 3 that $A$ has finite rank so that $G / A$ has infinite rank and hence it is a $T$-group by the minimal choice of $G$. In particular, $G^{\prime} / A$ is abelian and $A=G^{\prime \prime}$. Let $p$ be a prime number such that $A$ contains elements of order $p$, and let $A_{p^{\prime}}$ be the $p^{\prime}$-component of $A$. Then $G / A_{p^{\prime}}$ is likewise a counterexample, and hence without loss of generality we may suppose that $A_{p^{\prime}}=\{1\}$ and $A$ is a $p$-group. It follows that $G / C_{G}(A)$ is finite and $K=C_{G}(A)$ has infinite rank. 
As $K^{\prime} A / A$ is abelian, the subgroup $K^{\prime}$ is nilpotent, and it follows from Lemma 8 that $K$ is a $T$-group. Then $C_{K}\left(K^{\prime}\right)$ coincides with the Fitting subgroup of $K$, and it is a Dedekind group. On the other hand, the centralizer $C_{G^{\prime}}(A)$ is a nilpotent normal subgroup of $K$ so that $C_{G^{\prime}}(A)$ is also a Dedekind group. Since the index $\left|G^{\prime}: C_{G^{\prime}}(A)\right|$ is finite, it follows that $G^{\prime}$ contains an abelian subgroup of finite index. Then $G^{\prime}$ also contains an abelian $G$-invariant subgroup $B$ of finite index, and another application of Corollary 3 yields that $B$ has finite rank. For each prime number $q$, let $B_{q^{\prime}}$ be the $q^{\prime}$-component of $B$. Then $G^{\prime} / B_{q^{\prime}}$ is a Černikov group, and so $G / B_{q^{\prime}}$ is a $T$-group by Lemma 4. In particular, $G / B_{q^{\prime}}$ is metabelian so that $G^{\prime \prime}$ is contained in $B_{q^{\prime}}$ and hence $G$ is metabelian because

$$
\bigcap_{q} B_{q^{\prime}}=\{1\}
$$

This contradiction proves the theorem.

The following example shows that the statement of the main theorem becomes false for non-periodic groups, even in the metabelian case.

Let $A$ be the additive group of rational numbers, and for each prime number $p$ let $x_{p}$ be the automorphism of $A$ defined by setting $a x_{p}=p a$ for each element $a$ of $A$. Then

$$
X=\underset{p}{\operatorname{Dr}}\left\langle x_{p}\right\rangle
$$

is a free abelian subgroup of infinite rank of the full automorphism group of $A$, and the semidirect product

$$
G=X \ltimes A
$$

is a torsion-free metabelian group. Clearly, $A$ is a minimal normal subgroup of $G$, and so $G$ is not a $T$-group. On the other hand, it is easy to show that any subnormal subgroup of $G$ either is contained in $A$ or contains $A$; in particular, all subnormal subgroups of infinite rank of $G$ contain $A$ and hence they are normal in $G$.

As the above example is torsion-free, one could ask whether our main theorem could be generalized to arbitrary soluble groups, looking at the behaviour of periodic subnormal subgroups. However, our next example shows that there exist metabelian groups containing a periodic subnormal non-normal subgroup, but in which all subnormal subgroups of infinite rank are normal.

Let $p$ be an odd prime number, and let $P$ be a group of type $p^{\infty}$. If $U$ is the full automorphism group of $P$, the semidirect product $H=U \ltimes P$ is a soluble $T$-group of infinite rank. Now consider a cyclic group $\langle x\rangle$ of order $p$, and the direct product $G=H \times\langle x\rangle$. Then every subnormal subgroup of infinite rank of $G$ contains $P$, and so it is normal in $G$; on the other hand, if $a$ is an element of order $p$ of $P$, the finite subnormal subgroup $\langle a x\rangle$ is not normal in $G$. Note also that the group $G$ is residually $T$.

In order to prove Theorem B, we need the following classical result of Carin concerning normal subgroups of finite rank in locally nilpotent groups (see, for instance, [13, Part 2, Lemma 6.37).

Lemma 9. Let $G$ be a locally nilpotent group, and let $A$ be a torsion-free abelian normal subgroup of $G$ of finite rank $r$. Then $A$ is contained in the rth term $Z_{r}(G)$ of the 
upper central series of $G$, and the factor group $G / C_{G}(A)$ is a torsion-free nilpotent group of finite rank.

A group is said to be hypercentral if it coincides with the last term of its upper central series. Then any nilpotent group is hypercentral, and all hypercentral groups are locally nilpotent. Moreover, since in a group with torsion-free centre all factors of the upper central series are likewise torsion-free (see, for instance, [13, Part 1, Theorem 2.25]), it follows from Lemma 9 that in any locally nilpotent group $G$ each torsion-free nilpotent normal subgroup of finite rank is contained in some term with finite ordinal type of the upper central series of $G$.

LEMMA 10. Let $G$ be a torsion-free hypercentral group whose subnormal subgroups of infinite rank are normal. Then either $G$ has finite rank or it is a T-group.

Proof. Let $A$ be a maximal abelian normal subgroup of $G$. If $A$ has infinite rank, then the group $G$ has the property $T$ by Corollary 3. Suppose that $A$ has finite rank. As $C_{G}(A)=A$, it follows from Lemma 9 that $G / A$ has finite rank, and hence $G$ itself has finite rank.

Recall that the group $G$ is called a Fitting group if it is generated by nilpotent normal subgroups, or equivalently if $G$ coincides with its Fitting subgroup. Clearly, any Fitting group is locally nilpotent, and all its cyclic subgroups are subnormal.

LEMMA 11. Let $G$ be a Fitting group whose subnormal subgroups of infinite rank are normal. Then either $G$ has finite rank or it is a T-group.

Proof. Assume that the statement is false, and let $G$ be a counterexample. Then all nilpotent normal subgroups of $G$ have finite rank by Corollary 3. If $N$ is the subgroup of $G$ consisting of all elements of finite order, it follows from Lemma 9 that the torsionfree group $G / N$ is hypercentral, since it is generated by torsion-free nilpotent normal subgroups. Moreover, $G / N$ is a $T$-group, because if $N$ has infinite rank, the property $T$ is a direct consequence of the hypothesis, whereas if $N$ has finite rank, we can apply Lemma 10 to $G / N$. Therefore, the Fitting group $G / N$ is abelian, and hence $G^{\prime}$ is periodic. If $G^{\prime}$ has a infinite rank, it follows from Theorem A that it is a $T$-group; then $G^{\prime}$ is a Dedekind group, and so $G$ is a $T$-group by Corollary 3. This contradiction shows that $G^{\prime}$ has finite rank, and so $G / G^{\prime}$ has infinite rank.

Let $K$ be any $G$-invariant subgroup of finite index of $G^{\prime}$. Then $G / K$ is a nilpotent group of infinite rank so that it is a Dedekind group and hence $\left|G^{\prime}: K\right| \leq 2$. It follows that $G^{\prime}=P \times Q$, where $P$ is an infinite $p$-group and $Q$ is a $p^{\prime}$-group. Moreover, $P$ is nilpotent and satisfies the minimal condition on subgroups so that $P / Z(P)$ is finite (see [13] Part 1, Theorem 3.14) and there exists finite characteristic subgroup $P_{0}$ of $P$ such that $P / P_{0}$ is the direct product of $n$ subgroups of type $p^{\infty}$ for some positive integer $n$. The factor group $G / P_{0} Q$ is clearly a counterexample, and hence replacing $G$ by $G / P_{0} Q$ we may suppose without loss of generality that $G^{\prime}$ is the direct product of $n$ groups of type $p^{\infty}$. Let $X$ be any nilpotent normal subgroup of $G$. Then the product $X G^{\prime}$ is nilpotent, and so

$$
\left[G^{\prime}, \underset{n}{X, X} \underset{\longrightarrow}{\longrightarrow}\right]=\{1\}
$$

As $G$ is generated by its nilpotent normal subgroups, it follows that

$$
\left[G^{\prime}, \underset{n}{G, \ldots}, G\right]=\{1\}
$$


so that $G$ is nilpotent, and this last contradiction completes the proof of the lemma.

Our second main result is an almost obvious consequence of Lemma 11.

Proof of Theorem $B$. Let $F$ be the Fitting subgroup of $G$. Then $F$ is a Fitting group, and so it follows from Lemma 11 that $F$ is a $T$-group. In particular, $F$ is nilpotent, and hence $G$ is a $T$-group by Corollary 3 .

ACKNOWLEDGEMENTS. This work was partially supported by MIUR-PRIN 2009 (Teoria dei Gruppi e Applicazioni). The first three authors are members of GNSAGA (INdAM). The last author is grateful for the hospitality from the University of Napoli Federico II and for the financial support from the Istituto Nazionale di Alta Matematica during the preparation of this paper.

\section{REFERENCES}

1. M. De Falco, F. de Giovanni, C. Musella and Y. P. Sysak, On metahamiltonian groups of infinite rank, J. Algebra (to appear).

2. M. De Falco, F. de Giovanni, C. Musella and N. Trabelsi, Groups whose proper subgroups of infinite rank have finite conjugacy classes, Bull. Austral. Math. Soc. (to appear).

3. F. De Mari and F. de Giovanni, Groups satisfying the maximal condition on subnormal non-normal subgroups, Colloquium Math. 103 (2005), 85-98.

4. F. De Mari and F. de Giovanni, Groups satisfying the minimal condition on subnormal non-normal subgroups, Algebra Colloq. 13 (2006), 411-420.

5. M. R. Dixon, M. J. Evans and H. Smith, Locally soluble-by-finite groups of finite rank, J. Algebra 182 (1996), 756-769.

6. M. R. Dixon, M. J. Evans and H. Smith, Locally (soluble-by-finite) groups with all proper non-nilpotent subgroups of finite rank, J. Pure Appl. Algebra 135 (1999), 33-43.

7. M. R. Dixon, M. J. Evans and H. Smith, Groups with all proper subgroups (finite rank)-by-nilpotent, Arch. Math. (Basel) 72 (1999), 321-327.

8. M. R. Dixon and Z. Y. Karatas, Groups with all subgroups permutable or of finite rank, Centr. Eur. J. Math. 10 (2012), 950-957.

9. M. J. Evans and Y. Kim, On groups in which every subgroup of infinite rank is subnormal of bounded defect, Comm. Algebra 32 (2004), 2547-2557.

10. S. Franciosi and F. de Giovanni, Groups in which every infinite subnormal subgroup is normal, J. Algebra 96 (1985), 566-580.

11. W. Gaschütz, Gruppen in denen das Normalteilersein transitiv ist, J. Reine Angew. Math. 198 (1957), 87-92.

12. D. J. S. Robinson, Groups in which normality is a transitive relation, Proc. Camb. Philos. Soc. 68 (1964), 21-38.

13. D. J. S. Robinson, Finiteness conditions and generalized soluble groups (Springer, Berlin, Germany, 1972).

14. N. N. Semko and S. N. Kuchmenko: Groups with almost normal subgroups of infinite rank, Ukrain. Math. J. 57 (2005), 621-639. 